\begin{tabular}{|c|c|}
\hline Title & ELASTIC AND PLASTIC DEFORMATION OF CARBON NANOTUBES \\
\hline Author(s) & SHIMA, H.; SATO, M. \\
\hline Citation & $\begin{array}{l}\text { Proceedings of the Thirteenth East A sia Pacific Conference on Structural Engineering and Construction (EA SEC-13), } \\
\text { September 11-13, 2013, Sapporo, Japan, C-5-4., C-5-4 }\end{array}$ \\
\hline Issue Date & $2013-09-11$ \\
\hline Doc URL & http:/hdl .handle.net/2115/54292 \\
\hline Type & proceedings \\
\hline Note & $\begin{array}{l}\text { The Thirteenth East A sia Pacific Conference on Structural Engineering and Construction (EA SEC-13), September 11- } \\
\text { 13, 2013, Sapporo, Japan. }\end{array}$ \\
\hline File Information & easec13-C-5-4.pdf \\
\hline
\end{tabular}

Instructions for use 


\title{
ELASTIC AND PLASTIC DEFORMATION OF CARBON NANOTUBES
}

\author{
H. SHIMA ${ }^{1 *}$, and M. SATO ${ }^{2}$ \\ ${ }^{1}$ Department of Environmental Sciences, University of Yamanashi, Japan \\ ${ }^{2}$ Faculty of Engineering, Hokkaido University, Japan
}

\begin{abstract}
Can you imagine a material that is many times stronger than steel, yet flexible when bent or twisted, that withstands ultrahigh heat, and that conducts heat better than diamond? Does this dream material really exist? Yes. "Carbon nanotubes.” The excellent mechanical properties of nanotubes include an extremely high Young's modulus (ca. 1 TPa) and tensile strength (50 GPa or higher), whose values are several times higher than those of steel. Furthermore, since its specific gravity is less than one-tenth that of steel, it offers 100 times larger mechanical strength than steel with the same weight. Another characteristic of nanotubes is their extreme flexibility to bending and twisting deformation. It was experimentally confirmed that a nanotube returns to its original shape like a rubber hose even when the entire tube is twisted or bent until it is warped.

In this contribution, we will give a comprehensive overview of the research progress to date, focusing on the mechanical properties of carbon nanotubes. The first half examines minute deformations in the elastic region; the latter half takes a closer look at the plastic deformation of nanotubes under extreme conditions including giant deformation, high temperature and pressure and electron beam irradiation.
\end{abstract}

Keywords: Carbon nanotube, Buckling, High-energy beam irradiation, Thin-shell theory, Molecular dynamics simulation.

\section{INTRODUCTION}

Carbon is a rare substance which can take various structures and forms. When carbon atoms form a three-dimensional structure, their glittering beauty as diamonds captivates people. When aligned in a two-dimensional plane or hexagonal net, carbon atoms become just black graphite and lose their sparkle. In addition to these two macroscale carbon materials, a variety of nano-carbon materials have been discovered in just the last twenty years; graphene ribbons (Osawa 1970; Novoselov 2011) and peanut-shaped fullerene polymers (Onoe 2012) are only a few to mention.

\footnotetext{
* Corresponding author: Email: hshima@yamanashi.ac.jp
} 
Among the family of nano-carbon materials, carbon nanotubes (Iijima 1991) are attracting greatest attention both in industry and academia. Research on carbon nanotubes has focused on two characteristics not usually seen in other fields. First and foremost is the sheer breadth of the research, which encompasses physics, chemistry, materials science, electric and electronic engineering as well as life science. The second characteristic is that basic research and applied research are extremely close to each other. A succession of phenomena of interest to scientists has been discovered like a treasure chest, each leading to an innovative application or development. Thanks to these two characteristics, applied research extends as far as electronic devices, fuel cells, panel display materials and gas absorption and has seen dramatic progress.

One of the reasons why carbon nanotubes offer huge potential in so many fields is their excellent mechanical properties (Shima \& Sato 2013). Earlier studies have shown that mechanical deformation causes considerable changes in electronic, optical, magnetic and chemical properties. Thus, many studies on new technologies to utilize the correlation between deformation and properties are underway in various fields including electronics, biotechnology and material design (Shima 2013). For example, ultrahigh-strength materials are being developed for next-generation infrastructure such as skyscraper buildings and large aerospace equipment by utilizing the high thermal and environmental resistance of nanotubes. The application of these low-density substances containing only light carbon atoms for aircraft and automobile parts is expected to raise fuel efficiency and save energy, as well as dramatically reduce exhaust gas emissions and environmental impact.

\section{STRUCTURE OF CARBON NANOTUBES}

Carbon nanotubes are cylindrical substances comprising only carbon atoms. Their hollow centers are a vacuum, and they have fine structures of $1-10 \mathrm{~nm}$ in diameter and $1 \mu \mathrm{m}$ to $1 \mathrm{~mm}$ or more in length. As their diameters are smaller than the wavelength of visible light $(400-800 \mu \mathrm{m})$, they cannot be observed directly by a light microscope and so must be observed under larger, more expensive electron microscopes. The amazing robustness of nanotubes is solely due to chemical bonding between carbon atoms. The cylindrical sheet (graphite surface) which forms the nanotube has a seamless structure comprising a hexagonal grid of carbon atoms. The bonds between carbon atoms (known as "SP${ }^{2}$ carbons") that form hexagonal shapes such as benzene rings are the strongest of all atomic bonds. A carbon nanotube consists entirely of this strongest bond, and therefore, it is extremely resistant to bending or pulling, is very stable, and reacts with few chemicals.

\section{ELASTIC BUCKLING AND RESILIENCE}

To date, many intriguing post-buckling morphologies of nanotubes have been widely predicted in theory and observed in experiments (Shima 2012): local kinks in bent single-walled nanotubes (Iijima 1996), rippling or Yoshimura (diamond-shaped) patterns in tens-walled nanotubes (Arroyo 2003), and radial corrugation patterns under axially symmetric load (Shima et al. 2010; Shima et al. 
2013). One of the most outstanding features of post-buckling nanotubes is their remarkable buckling capacity. Indeed, experimental investigations have shown that the buckling deformation can be completely recovered when the load is removed. The marked resilience is primary because of: i) the large in-plane rigidity of grapheme sheets rather than relatively low bending rigidity, and ii) the intrinsic hollow geometry with extremely large aspect ratio that carbon nanotubes exhibit.
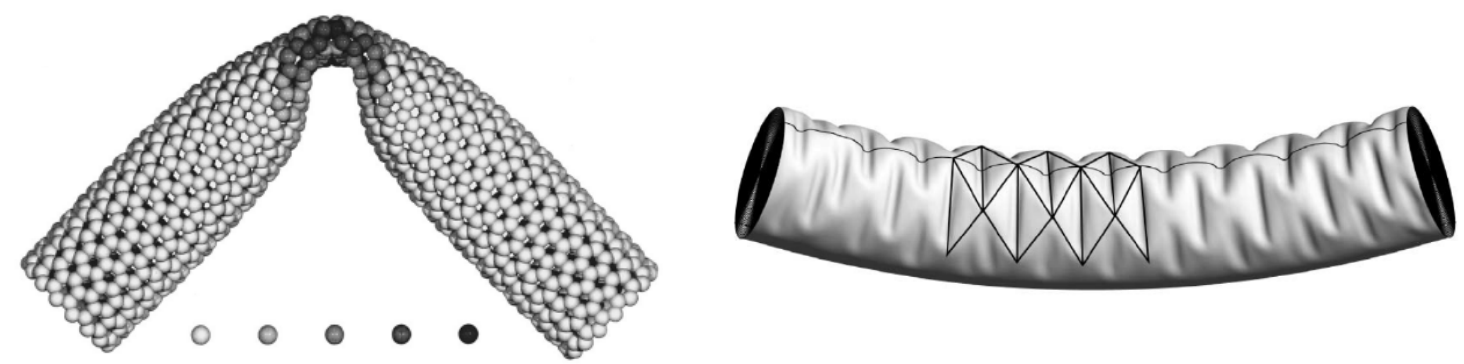

Figure 1: (Left) Kink structure formed in a single-walled nanotube under bending (Iijima 1996). (Right) Morphology of a rippled multi-walled nanotube reminiscent of the Yoshimura pattern. The ridges and furrows are highlighted, as well as the trace of the longitudinal section (Arroyo 2003).

Apart from the resilience, physical and mechanical properties of carbon nanotube are susceptible to buckling; namely, the breakdown of the structural symmetry due to the buckling may trigger sudden changes in physical properties. Besides, the buckling-induced reduction in nanotubes stiffness will not only impair their ability to sustain external loadings as reinforced fibers in nanocomposites but it also gives rise to large uncertainties in the vibration behavior of nanotubes as nanoscale resonators. These buckling-property relations can significantly influence the performance of nanotubes as structural or functional elements, thus being the primary reason why huge amount of effort has been paid to the study of nanotube bucklings.

\section{IRRADIATION-BASED TAIRLORING}

It is known that high-energy beam irradiation to carbon nanotubes leads to their plastic deformation, thus having beneficial effects in manipulating the carbon nanotube morphology, especially when combined with heat treatment (Krasheninnikov 2010). The manipulation is achieved through the irradiation-induced displacement of carbon atoms from their equilibrium positions. When the kinetic energy transferred from incident particles to the carbon nuclei is larger than the displace threshold, a carbon atom leaves its initial position and pushed far away from the nanotube or be quenched into a metastable defect configuration. Hence, bombardment with energetic particles can be used for the sputtering of atoms in predefined areas, and successive sputtering treatment makes it possible to alter the local (or larger-scale) geometry of nanotubes. 
(a)

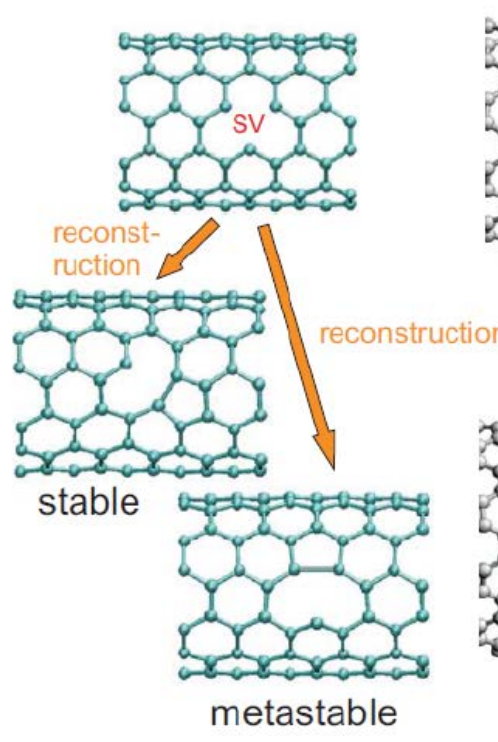

(b)

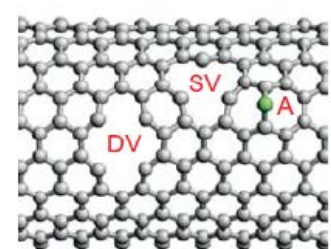

(c)

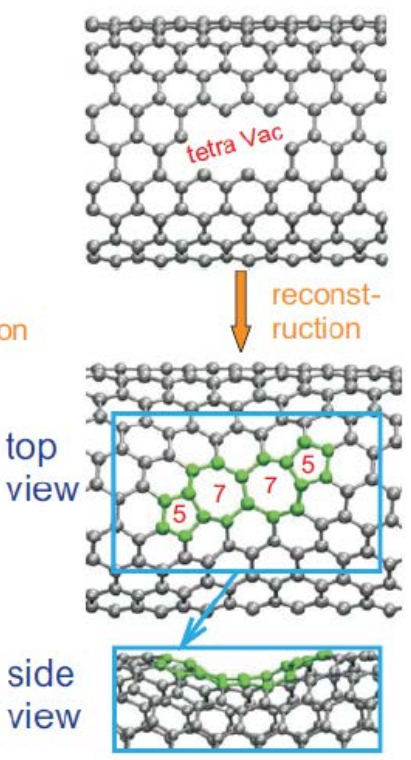

Figure 2: Reconstruction of the atomic network near vacancies produced by electron-beam irradiation. (a) A single vacancy (SV) state tends toward stable or metastable configurations depending on the orientation of the new weak pentagon bond. (b) A divacancy (DV) transforms to an agglomeration of five- and eight-membered rings. A SV and an adatom may form a metastable 5-7-7-5 defect. (c) Reconstruction of a tetravacancy. The effective size of the "hole" decreases at the expense of local diameter reduction (Krasheninnikov 2010).

In view of irradiation-based tailoring, vacancies are the most prolific defects in nanotubes. A vacancy originates from missing atoms in the ideal crystalline lattice. In usual solids, it keeps its void structure without curing as time passes; however, it is not the case in carbon nanotubes. The removal of carbon atoms from the purely hexagonal lattice leads to a local reconstruction so as to maintain its coherent network structure with cylindrical geometry. From this reason, carbon nanotubes are often referred to as "self-healing” materials.

The reconstruction procedure near vacancies is shown in Fig. 2. The panel (a) tells us that there are two choices of reconstruction of a single vacancy (SV) in an armchair carbon nanotube. Namely, the original unstable configuration (top panel) transforms into stable or metastable states by saturating two of the three dangling bonds around the vacancy. The latter two states are distinguished by the orientation of the "pentagon bond" that newly emerges near the missing atom. The final state should be metastable if the bond is parallel to the tube axis, or stable when the bond is almost perpendicular to the tube axis. The panel (b) depicts a reconstruction associated with three different kinds of small vacancies. A divacancy (DV) transforms to an agglomeration of five- and eight-membered rings, where the orientation of the newly emerging pentagon bonds is uniquely determined (i.e., perpendicular to the missing C-C bond) in contrast with the panel (a). The panel 
(b) also shows the possibility that a pair of one SV and one adatom forms a metastable 5-7-7-5 defect, which may occur depending on the relative position of the neighboring SV and adatom. The panel (c) shows reconstruction of a tetravacancy. The salient feature is that the effective size of the "hole" decreases (see top view) at the expense of local diameter reduction (side view). This vacancy-induced reduction in local diameter implies that, if many large-scale vacancies are dispersed intentionally, then we will observe a global diameter reduction over the whole tube surface damaged (Shima et al. 2010).

\section{CHALLENGE AND FUTURE DIRECTIONS}

In the present work, we have provided a bird's-eye view on the current state of knowledge on the non-trivial mechanical properties of carbon nanotubes. The understanding remains far from complete, but new experiments and theoretical work will no doubt give us a more complete picture and exciting times for both basic and applied research in the realm of nanoscale. Described below are two examples of challenging subjects that may trigger innovation in the relevant community.

\subsection{Buckling Effects on Heat Transport}

Carbon nanotubes demonstrate the excellent thermal conductivity among any known material. When a carbon nanotube is buckled, however, the localized structural deformation can prohibit ballistic heat transport along the nanotube axis. This results in the decreasing behavior of thermal conductivity of nanotubes under compressive stress, which is attributed to the increase in the phonon-phonon scattering rate. Such the buckling-induced reduction in thermal conductivity has important implications of heat management of nano-scale electronic devices, including the dynamic control of thermal transport and energy conversion. In view of materials sciences, it is also important to make clear the effect of buckling on the thermal properties of carbon nanotube composites. Despite this great potential, very limited number of studies has been conducted on the issue thus far.

\subsection{Relevance to Chemical Reaction}

Applications of carbon nanotubes in composite materials often require an understanding and control of the chemistry and chemical reactivity of the nanotubes' sidewall. This is because the carbon-carbon bonding state on the outermost graphitic surfaces determines where the chemically sensitive reactions take place and how the reactions affect the physical properties of carbon nanotubes. For instance, the functionalization and/or chemisorption on the sidewall of nanotubes enable to increase linking between nanotubes as filler and a surrounding matrix. Besides, reactivity control of the nanotube sidewall leads to a novel technique for chemical sensors and drug delivery systems. An important finding in the context of buckling is that the chemical reactivity of carbon nanotube is dependent on local surface curvature of the outermost sidewall. In fact, the reactivity of a nanotube is governed by the local atomic structure of carbon atoms on which the chemisorbing species or functional group can react and/or a stable bond. This result implies the control of 
sidewall reactivity by artificial deformation; that is, local chemical reactivity of carbon nanotubes can be promoted locally by inducing mechanical deformation.

\section{ACKNOWLEDGEMENT}

We cordially thank Yoshitaka Umeno at University of Tokyo, Marino Arroyo at Universitat Politecnita de Catalunya, and Susanta Ghosh at University of Michigan for illuminating discussions. Technical assistances by Yuta Ishiwata, Ayako Kusano, and Hisao Taira are also greatly acknowledged. This work was supported by JSPS KAKENHI (Grant Numbers 24686096, 11J03636, 22760058). H.S appreciates the financial support provided by the Asahi Grass Foundation.

\section{REFERENCES}

Arroyo M and Belytschko T (2003) Nonlinear Mechanical Response and Rippling of Thick Multiwalled Carbon Nanotubes. Phys. Rev. Lett. 91, 215505 [4 pages].

Iijima S (1991) Helical microtubules of graphitic carbon, Nature 354, pp.56-58.

Iijima S, Brabec C, Maiti A, and Bernholc J (1996) Structural flexibility of carbon nanotubes. J. Chem. Phys. 104, pp. 2089-2092.

Krasheninnikov A V and Nordlund K (2010) Ion and electron irradiation-induced effects in nanostructured materials. J. Appl. Phys. 107, 071301 [70 pages].

Onoe J, Ito T, Shima H, Yoshioka H, and Kimura S (2012) Observation of Riemannian geometric effects on electronic states. EPL (Europhys. Lett.) 98, 27001 [5pages].

Osawa E (1970) Superaromaticity. Kagaku 25, pp.854-863.

Shima H (2012) Buckling of Carbon Nanotubes: A State of the Art Review. Materials. 5, pp.47-84.

Shima H (2013) Geometry-property relation in corrugated nanocarbon cylinders, in "Modeling of Carbon Nanotubes, Graphene and Their Composites” edited by K. Tserpes and N. Silvestre. Springer-Verlag.

Shima H, Sato M, Iiboshi K, Ghosh S, and Arroyo M (2010) Diverse corrugation pattern in radially shrinking carbon nanotubes. Phys. Rev. B 82, 085401 [7pages].

Shima H and Sato M (2013) Elastic and Plastic Deformation of Carbon Nanotubes. Pan Stanford Publishing. ISBN: 978-981431075-8.

Shima H, Umeno Y and Sato M (2013) Molecular dynamics study of radial corrugation in carbon nanotubes. Mech. Adv. Mater. Str. in press. 